gestartet wurde und Patienten zum Zeitpunkt der Diagnose häufiger fortgeschrittene Tumoren hatten als heutzutage. $\mathrm{Zu}$ dem haben verbesserte Operationstechniken zu einer kürzeren Operationsdauer und Krankenhausverweildauer geführt. Für neue Methoden wie die robotergestütze laparoskopische Prostatektomie liegen derzeit noch keine Daten über
Langzeitkosten vor. Die Autoren vermuten, dass diese die der klassichen RP noch übersteigen.

Fazit: Eine radikale Prostatektomie inklusive aller Nachbehandlungen verursacht etwa um $34 \%$ höhere Kosten als Watchful Waiting. Dieser Unterschied, der fast ausschließlich auf die Kosten des
Eingriffs zurückzuführen sind, lässt sich im Laufe des Follow-up nicht mehr aufholen.

slx

Andersson SO et al. Managing localized prostate cancer by radical prostatectomy or watchful waiting: Cost analysis of a randomized trial (SPCG-4). Scand J Urol Nephrol. 2011; 45: 177-83

\section{Nierenteilresektion versus radikale Nephrektomie}

Eine Nierenteilresektion ist eine sichere Methode, um lokale Nierenzellkarzinome zu behandeln, mit einer etwas höheren Komplikationsrate als bei einer radikalen Nephrektomie. In einer internationalen Multicenterstudie wurde untersucht, inwiefern sich das onkologische Outcome der beiden Methoden unterscheidet.

$\mathrm{D}$ ie Nierenteilresektion (PN) gilt bei Nierenzellkarzinomen (RCC) im Stadium T1a $(<4 \mathrm{~cm})$ und gesunder kontralateraler Niere als Standardtherapie. Die Empfehlung, organerhaltend zu operieren, basiert auf Studien, in denen die onkologischen Ergebnisse unter NTR ähnlich gut waren wie nach einer radikalen Nephrektomie (RN), bei gleichzeitigem Erhalt der Nierenfunktion. Keine der bisherigen Vergleichsstudien war jedoch randomisiert.

1992 wurde eine internationale Multicenterstudie mit dem Ziel gestartet, Morbidität und Tumorkontrolle unter den beiden Operationsmethoden zu vergleichen. 541 Patienten mit Tumorstadium T1-T2 N0 M0 aus 17 Ländern wurden entweder einer Nierenteilresektion ( $\mathrm{n}=$ 268) oder einer radikalen Nephrektomie $(\mathrm{n}=273)$ zugeteilt. 55 Patienten wechselten die Behandlung: 16 der RN-Patienten $(5,9 \%)$ wollten auf eigenen Wunsch eine $\mathrm{RN}$, bei 39 der RN-Patienten (14,6\%) musste aus pathologischen Gründen eine $\mathrm{RN}$ durchgeführt werden.

Beide Methoden sind sicher, unter PN war die Komplikationsrate etwas höher (z.B. perioperative Blutungen als häufigste
Komplikation $28,3 \%$ vs. $17,5 \%$ ). Insgesamt verstarben nur 12 Patienten (2,2\%) tumorbedingt ( 4 in der RN-Gruppe, 8 in der PNGruppe). Aufgrund der wenigen Todesfälle war das Sterberisiko zwischen den beiden Methoden nicht signifikant. Die häufigsten Todesursachen waren kardiovaskuläre Ereignisse (9,3\% in der PN-Gruppe,
7,3\% in der RN-Gruppe). Auch das 10-Jahres-Überleben unterschied sich nicht signifikant $(75,7 \%$ bei PN, $81,1 \%$ bei $R N)$.

Fazit: Mit beiden Methoden lassen sich hervorragende onkologische Ergebnisse erzielen. Um eine Übertherapie zu vermeiden, sollte wenn möglich auf die organerhaltende Technik zurückgegriffen werden, so die Autoren.

van Poppel $\mathrm{H}$ et al. A prospective, randomised EORTC intergroup phase 3 study comparing the oncologic outcome of elective nephron-sparing surgery and radical nephrectomy for low-stage renal cell carcinoma. Eur Urol 2011; 59:543-52

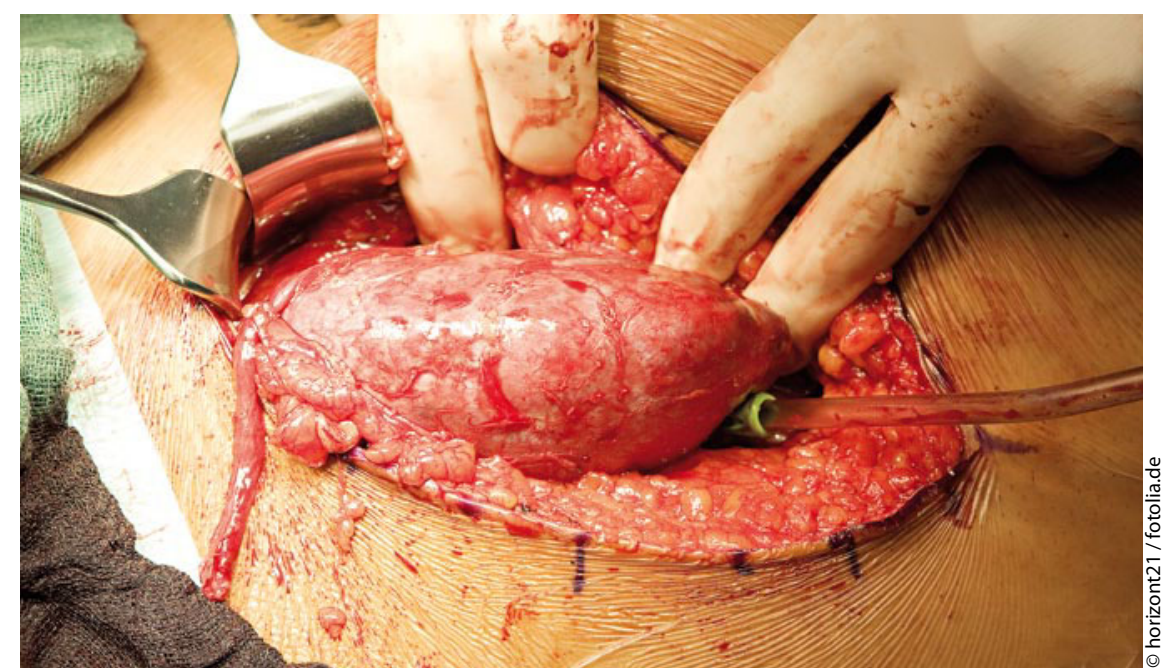

Nierenteilresektion und radikale Nephrektomie sind beides sichere Methoden zur Therapie eines Nierenzellkarzinoms. Doch wie unterscheidet sich das onkologische Outcome? 